\title{
Assessment of awareness of post-hospital rehabilitation need in patients after acute coronary syndrome and after stroke
}

\author{
Dominika Szalewska', Agnieszka Dudaniec-Tarkowska', Piotr Zieliński² \\ ${ }^{1}$ Department of Rehabilitation, Medical University of Gdańsk, Poland \\ ${ }^{2}$ Department of Sports Medicine, University of Physical Education and Sport in Gdańsk, Poland
}

Szalewska D, Dudaniec-Tarkowska A, Zieliński P. Assessment of awareness of post-hospital rehabilitation need in patients after acute coronary syndrome and after stroke. Ann Agric Environ Med. 2017; 24(3): 537-540. doi: 10.5604/12321966.1233981

\begin{abstract}
Introduction. Acute coronary syndrome (ACS) and stroke are the leading causes of mortality and long-term morbidity across the world. Post-hospital rehabilitation (PHR) is strongly recommended in both groups.

Objective. The purpose was assessment of awareness of the PHR need in patients after ACS and after ischaemic stroke (IS). Materials and method. The study included 60 patients $(17 \mathrm{~F}, 43 \mathrm{M}), 62 \pm 13.6$ years of age, admitted to hospital due to ACS ( $n=30$ pts) or IS ( $n=30$ pts). A cross-sectional survey was carried out in order to evaluate awareness of the PHR need through a questionnaire composed of 14 one-choice answer questions.

Results. There was no significant correlation between place of residence and willingness to participate in the PHR programme: $69.2 \%(n=9)$ of the pts living in rural areas and $80.9 \%(n=38)$ living in the city declared their willingness to partcipate. The majority of patients declared that PHR is needed ( $87.5 \%$ of pts with basic educational level, $63.2 \%$ with vocational education, $61.1 \%$ with technical education, $80 \%$ with university education level). $43.3 \%(n=13)$ of pts after ACS and $66.7 \%(n=20)$ pts after IS declared they had been informed about the possibility of PHR. Altogether, $46.7 \%(n=14)$ of pts after ACS and $33.3 \%$ $(n=10)$ after stroke understood it to be a stay in a spa; only $10 \%(n=3)$ of pts after ACS and $33.3 \%(n=10)$ after IS understood it correctly. 30\% $(n=9)$ pts after ACS and $13.3 \%(n=4)$ after IS saw it as a change in lifestyle and $13.3 \%(n=4)$ pts after ACS and $20 \%(n=6)$ after IS declared they did not know what PHR was.

Conclusions. Type of place of residence and educational level had no association with the PHR need. Patients after IS were more aware than those after ACS. Increased independence was the main expectation in both groups. The majority of patients perceived PHR to consist of treatment in a spa. Too few patients were informed about the possibility of PHR.
\end{abstract}

\section{Key words}

rehabilitation, acute coronary syndrome, stroke

\section{INTRODUCTION}

Acute coronary syndrome (ACS) and stroke are the leading causes of mortality worldwide [1,2]. Ischaemic heart disease and cerebrovascular disease also result in substantial longterm morbidity, and are the uppermost causes of the overall disease burden, measured in disability-adjusted life-years [DALYs] lost.[3] Post-hospital rehabilitation is strongly recommended in both groups of patients. Secondary prevention through cardiac or neurological rehabilitation is of paramount importance since ischaemic events continue to augment at a high rate after the acute phase. In a database of $16,321 \mathrm{ACS}, 20 \%$ of all patients were re-hospitalized and $18 \%$ of men and $23 \%$ of women $>40$ years of age died during the first year following an acute ischaemic event [4]. Cardiac rehabilitation is recommended by the European Society of Cardiology in all patients after ACS, and similarly, according to the guidelines of the European Stroke Organisation, rehabilitation after stroke is also strongly recommended in all patients (I class, level A of recommendations) [5,6,7,8]. Metaanalysis performed in the era before reperfusion therapy showed that cardiac rehabilitation programmes

Address for correspondence: Dominika Szalewska, Department of Rehabilitation, Medical University of Gdańsk

E-mail:dziel@gumed.edu.pl

Received: 21 November 2013; accepted: 27 June 2014; first published:February 2017 including exercise training decreased the mortality index in patients with coronary artery disease by $26 \%[9,10]$. The programmes should be introduced as quickly as possible after admission to hospital and continued in the following weeks and months. They should be multidisciplinary and focused on minimizing the coronary artery disease risk factors. One of the goals of environmental medicine is to develop and implement short-and long-term plans for health promotion and health education. Patients' awareness, defined as a state or ability to perceive or to be conscious of events, is necessary to improve compliance and the effects of comprehensive pharmacological and non-pharmacological treatment in patients after ACS or stroke.

\section{OBJECTIVE}

The primary aim of the study was evaluation of the awareness of the need for post-hospital rehabilitation in patients after acute coronary syndrome and after stroke. The secondary purpose was the assessment of factors influencing it.

The following detailed research questions were formulated. 1. Is there any association between place of residence (urban or rural area) and the willingness to participate in posthospital rehabilitation programmes?

2. Is there a relationship between professional activity and the type of illness? 
3. Is there an association between education level and consciousness of the need for post-hospital rehabilitation in patients after ACS and after stroke?

4. Is there a relationship between physical activity and type of illness?

5. Were patients informed about post-hospital rehabilitation during the hospital stay?

6. What are the patients' expectations about the effects of post-hospital rehabilitation?

7. What are the patients' understanding of post-hospital rehabilitation?

8. Are there any significant differences in the consciousness of the need for post-hospital rehabilitation in patients after ACS and after stroke?

9. Are there any significant differences in the willingness to participate in post-hospital rehabilitation in patients after ACS and after stroke?

\section{MATERIALS AND METHOD}

Study population. A total of 60 consecutive, eligible patients (17 females, 43 males), $62 \pm 13.6$ years of age, admitted to the Department of Cardiology due to ACS and admitted to the Department of Neurology due to ischaemic stroke were included in the study. Of these, 30 patients $(58 \pm 13.5$ years of age) were hospitalized due to ACS and 30 (66 \pm 12.6 years of age) were hospitalized due to ischaemic stroke. The study was conducted in the period 14 November-14 December 2012.

Inclusion criteria for the study included hospitalization due to acute coronary syndrome, defined as unstable angina or acute myocardial infarction in patients presenting with ST-segment elevation (STEMI), acute coronary syndrome in patients presenting without persistent ST-segment elevation (NSTEMI) and ischaemic stroke. The exclusion criteria were aphasia and lack of consent to participate in the study.

The 'cardiac' group included three women $(10 \%$ of the study population), while the 'neurological' group included 14 women (47\%).

The reason for hospitalization in the Cardiology Department was ACS was presented in $80 \%$ as unstable angina $(n=24)$ and in $20 \%$ as myocardial infarction $(n=6)$. The reason for hospitalization in the Department of Neurology was ischaemic stroke.

Questionnaire. The tool used in this cross-sectional study was a questionnaire composed of 14 one-choice answer questions. The first part featured information gathered by the author concerning age, gender, and current hospitalization details; the questions in the second part concerned physical activity, place of residence, and awareness of the need for post-hospital rehabilitation. After signing agreement to participate in the study, the patients answered questions asked by and explained by the author.

Statistics. SPSS Statistics version 20 was used for statistics. Means, standard deviations and percent were used to summarize the characteristics of the study population. Quantitative parameters were compared using paired Student's test. The Chi-square test was used to compare the results of the survey between both groups. Values of $\mathrm{p}<0.05$ were assumed as statistically significant.

\section{RESULTS}

Characteristics of the study population. Baseline characteristics of patients in both groups are outlined in Table 1. Patients after ACS were younger than patients after stroke, and there were more male patients than in the group after stroke. Co-morbidities were as follows: arterial hypertension ( $48.3 \% ; \mathrm{n}=29)$, myocardial infarction peractus (31.7\%; $n=19)$, diabetes mellitus t. $2(18.3 \%$; $=11)$ and a history of cancer in one patient.

A total of $78.4 \%$ of all patients $(n=47)$ lived in cities while $21.6 \%(n=13)$ lived in rural areas. In the 'cardiac' group, $76.7 \%$ $(n=23)$ of patients lived in cities while $23.3 \%(n=7)$ came from rural areas. In the 'neurological' group, only $20 \%(n=6)$ came from rural areas.

A total of $36.7 \%(n=22)$ of patients were current smokers or had a history of smoking in the past five years; $63.3 \%$ of patients $(n=38)$ had never smoked.

Of all patients, $13.3 \%(n=8)$ had only a basic level of education, $31.6 \%(n=19)$ had vocational education, $30 \%$ $(n=18)$ had a technical education level and $25 \%(n=15)$ had a university degree. In the 'cardiac' group, the education levels were as follows: $10 \%(n=3), 40 \%(n=12), 30 \%(n=9)$ and $20 \%$ $(n=6)$, respectively. In patients after stroke, $16.7 \%(n=5)$ had a basic education level, 23.3\% ( $n=7)$ vocational education, 30\% $(n=9)$ technical education, and 30\% $(n=9)$ had a university degree. Of the patients, $46.7 \%(n=14)$ from the Department of Cardiology and 26.7\% $(n=8)$ from the Department of Neurology were professionally active. Professional activity was similar in both groups $\left(\mathrm{chi}^{2}=2.58 ; \mathrm{p}>0.05\right)$.

Table 1. Characteristics of patients after acute coronary syndrome and patients after ischaemic stroke

\begin{tabular}{lccc}
\hline Characteristics & $\begin{array}{c}\text { Patients after } \\
\text { ACS } \\
(\mathrm{n}=30)\end{array}$ & $\begin{array}{c}\text { Patients after } \\
\text { ischaemic stroke } \\
(\mathrm{n}=30)\end{array}$ & $\mathrm{p}$ \\
\hline Age-years & $58 \pm 13,5$ & $66 \pm 12,6$ & $<0,05$ \\
\hline Male gender, $\mathrm{n}(\%)$ & $27(90)$ & $16(53)$ & $<0,05$ \\
\hline Hypertension, $\mathrm{n}(\%)$ & $13(43)$ & $16(53)$ & $\mathrm{NS}$ \\
\hline Smoking habit, $\mathrm{n}(\%)$ & $11(37)$ & $12(39)$ & $\mathrm{NS}$ \\
\hline Physical activity & $17(57)$ & $14(47)$ & $\mathrm{NS}$ \\
$\geq 3$ times a week, $\mathrm{n}(\%)$ & $14(47)$ & $8(27)$ & $\mathrm{NS}$ \\
\hline Professional activity & $7(23)$ & $6(20)$ & $\mathrm{NS}$ \\
\hline Place of residence: rural areas, $\mathrm{n}(\%)$ & & &
\end{tabular}

Plus-minus values are means \pm SD

Questionnaire outcomes. There was no significant correlation between place of residence and willingness to participate in the post-hospital rehabilitation programme: $69.2 \%(n=9)$ of patients living in rural areas and $80.9 \%(n=38)$ living in the city declared their willingness to participate in the post-hospital rehabilitation programme $\left(\mathrm{chi}^{2}=0.81\right.$; $\mathrm{p}>0.05$ ) (Fig. 1).

Education level did not significantly correlate with awareness of the need for post-hospital rehabilitation. The majority of patients, with no difference in level of education, declared that post-hospital rehabilitation is needed $(87.5 \%$ of patients characterized by basic educational level, $63.2 \%$ with vocational education, $61.1 \%$ with technical education and $80 \%$ with university education level). 


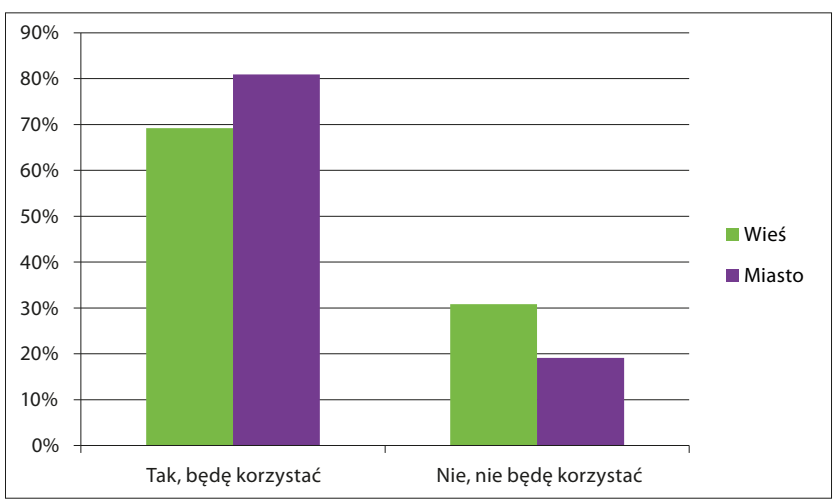

Figure 1. Relation between place of residence and willingness to participate in the post-hospital rehabilitation programme

All patients from the 'cardiac' group declared they were physically active: $43.3 \%(n=13)$ of patients said they were active at least $1-2$ times/week and $56.7 \%$ of patients $3-5$ times/week. Among patients after stroke, $90 \%$ of patients $(\mathrm{n}=27)$ declared themselves to be physically active: respectively, $48.1 \%(n=13)$ and $51.9 \%(n=14) 1-2$ times/week and 3-5 times/week. Physical fitness did not differ among the groups $\left(\mathrm{chi}^{2}=3.15, \mathrm{p}>0.05\right)$.

Only $43.3 \%(n=13)$ of patients after ACS and $66.7 \%(n=20)$ patients after stroke declared they had been informed about the possibility of continued hospital rehabilitation in the form of post-hospital forms of physiotherapy; the statistical difference between the numbers of patients informed and not informed was borderline $\left(\mathrm{chi}^{2}=3.30 ; \mathrm{p}=0.059\right)$.

Expectations in relation to post-hospital rehabilitation varied greatly (Fig. 2). Among patients from the Cardiology Department, $43.3 \%(n=13)$ expected rehabilitation to improve their independence in daily living, 30\% $(n=9)$ expected full recovery, $20 \%(n=6)$ expected to prolong life, and $6.7 \%(n=2)$ expected reduction in pain. Patients from the Department of Neurology expected, respectively: $46.7 \%(n=14)$ improvement of independence in everyday life, $30 \%(n=9)$ full recovery,

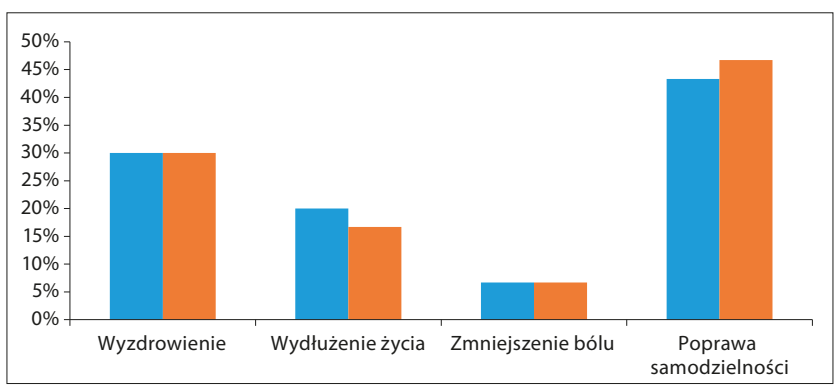

$16.7 \%(n=5)$ prolonged life and $6.7 \%(n=2)$ reduction in pain.

Figure 2. Patients' expectations about the effects of post-hospital rehabilitation

Patients' understanding of post-hospital rehabilitation is illustrated in Figure 3: 46.7\% $(n=14)$ of patients after ACS and $33.3 \%(n=10)$ of patients after stroke understood posthospital rehabilitation as a stay in a spa; only $10 \%(n=3)$ patients after ACS and 33.3\% $(n=10)$ after stroke understood it correctly as a comprehensive programme in the Department of Rehabilitation. A total of $30 \%(n=9)$ patients after ACS and $13.3 \%(n=4$ patients) after stroke saw it as a change in lifestyle, while $13.3 \%(n=4)$ from the Cardiology Department and $20 \%(n=6)$ from the Neurology Department declared that they did not know what post-hospital rehabilitation was.

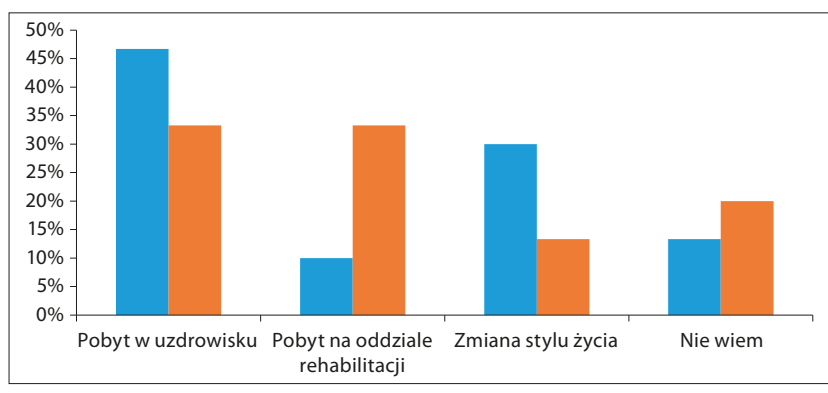

Figure 3. Patients' understanding of post-hospital rehabilitation

Significantly more patients after stroke $(83.3 \% ; n=25)$ than after ACS $(56.7 \% ; n=17)$ answered that post-hospital rehabilitation programmes are necessary $\left(\mathrm{chi}^{2}=5.08 ; \mathrm{p}<0.05\right)$. Similarly, significantly more patients after stroke (93.3\%; $\mathrm{n}=28)$ than after ACS (63.3\%; $\mathrm{n}=19)$ declared willingness to participate in a post-hospital rehabilitation programme $\left(\mathrm{chi}^{2}=7.95 ; \mathrm{p}<0.05\right)$.

\section{DISCUSSION}

All patients with ACS or after stroke should be enrolled in post-hospital rehabilitation programmes, but it is for the patients themselves to decide to decide whether or not to participate in the treatment. The presented study proves that patients after stroke are more aware of the need for posthospital rehabilitation than patients after acute coronary syndrome, and that 5 increased independence in everyday living was the main expectation in both groups of patients. Only 3 of 30 patients after ACS (3\%) and 10 of 30 (33.3\%) patients after stroke understood correctly what post-hospital rehabilitation is - that it is a comprehensive programme led by the Rehabilitation Department. The majority of patients perceived post-hospital rehabilitation to consist of treatment in a spa. Only $63.3 \%$ of patients after ACS declared willingness to participate in a post-hospital rehabilitation programme.

It has been proved that implementation of secondary prevention through cardiac rehabilitation survives life in at least the same number of patients as invasive treatment in the acute phase of ACS $[11,12]$, and that not referring a patient to the Rehabilitation Department is malpractice. The effects of exercise training in patients with heart failure, the number of which is increasing with the progress of medicine, should also not be underestimated [13].

Enrolment in a post-hospital cardiac rehabilitation/ secondary prevention programme can enhance patient compliance with the medical regimen, and is particularly advised for those with multiple modifiable risk factors and moderate-to-high risk patients for whom supervised guidance is warranted [14]. Exercise-based rehabilitation has been shown to be effective at reducing all-cause mortality and the risk of re-infarction, as well as diminishing risk factors, exercise-based capacity and health-related quality of life after myocardial infarction $[15,16]$.

Exercise therapy has long been used for rehabilitation purposes following myocardial infarctions, and the benefit of regular physical exercise in stable CAD patients is also well established. It can reduce the anxiety associated with the life-threatening illness and improve patient self-confidence. Mechanisms considered to be important mediators of a reduced cardiac event rate are: improvement of endothelial 
function, reduced progression of coronary lesions, reduced thrombogenic risk and improved collateralization. In a large meta-analysis, exercise training as part of coronary rehabilitation programmes was associated with a reduction of $26 \%$ in the cardiac mortality rate in patients with CAD [17].

The aim of post-hospital rehabilitation in patients after stroke is more complex than in patients after ACS bnecause, even with optimal stroke unit care including thrombolysis, less than one third of patients recover fully from stroke [18], and the goals of rehabilitation can shift from initial input to minimize impairment to more complex interventions designed to encourage active participation and prevent future events. Rehabilitation aims not only to prolong life, improve exercise tolerance and prevent further incidents, but also to enable the patient to reach and maintain optimal intellectual, psychological and/or social function [19]. One of the most important findings of the presented study is that less than half of a group of patients after ACS, and slightly more than half of a group of patients after stroke, were advised about rehabilitation. Evidence concerning the quality of secondary prevention of coronary artery disease (CAD) in Poland in recent years is scarce. Jankowski et al. showed modest improvement in the implementation of CAD secondary prevention guidelines in everyday clinical practice between 2006-2007 and 2011-2012 in the meaning of the better control of blood pressure in the population from 5 Polish hospitals, although the control of all other main risk factors did not change significantly [20]. Early initiation of rehabilitation after stroke is strongly recommended and early discharge from a Stroke Unit care is possible in medically stable patients with mild or moderate impairment, providing that post-hospital rehabilitation is delivered by a multidisciplinary team with stroke expertise.

Details concerning cardiac rehabilitation programmes are described in the document prepared by the ESC Working Group ESC [21], and information concerning neurological rehabilitation can be found in the guidelines of the European Stroke Organisation [5].

There is a strong need for improvement in the awareness of patients with coronary artery diseases and cerebrovascular diseases about post-hospital rehabilitation. Patients should be better informed about the effects of this treatment and understand that it is not a 'spa' which aims only at the improvement of quality of life, but is a method that decreases the risk of re-hospitalization and prolongs life.

\section{CONCLUSIONS}

1. There is no association between the place of residence and level of education and awareness of the need for posthospital rehabilitation.

2. Patients after stroke were more aware of the post-hospital rehabilitation need than patients after acute coronary syndromes.

3. Increased independence in everyday living was the main expectation in patients of both groups.

4. The majority of patients perceived post-hospital rehabilitation to consist of treatment in a spa.

5. Too few patients from both groups were informed about the possibility of post-hospital rehabilitation.

\section{REFERENCES}

1. Lopez AD, Mathers CD, Ezzati M, Jamison DT, Murray CJ. Global and regional burden of disease and risk factors: 2001: systematic analysis of population health data. Lancet 2006;367:1747-1757.

2. Sidney C. Smith, Jr. Reducing the global burden of ischemic heart disease and stroke: a challenge for the cardiovascular community and the United Nations. Circulation 2011;124:278-279.

3. Mathers CD, Loncar D. Projections of global mortality and burden of disease from 2002 to 2030. PLoS Med 2006;3:e442.

4. Menzin J, Wygant G, Hauch O, Jackel J, Friedman M. One-year costs of ischemic heart disease among patients with acute coronary syndromes: findings from a multi-employer claims database. Curr Med Res Opin $2008 ; 24: 461-468$.

5. European Stroke Organisation (ESO) Executive Committee: ESO Writing Committee. Cerebrovasc Dis 2008;25(5):457-507. doi: $10.1159 / 000131083$. Epub 2008 May. Guidelines for management of ischaemic stroke and transient ischaemic attack 2008.

6. Smith SC Jr, Allen J., Blair SN et al. AHA/ACC guidelines for secondary prevention for patients with coronary and other atherosclerotic vascular disease: 2006 update: endorsed by the National Heart, Lung, and Blood Institute. Circulation 2006;113: 2363-2372.

7. Bassand JP, Hamm CW, Ardissino D et al. Guidelines for the diagnosis and treatment of non-ST-segment elevation acute coronary syndromes. Eur Heart J 2007;28: 1598-1660.

8. Steg G, James SK, Atar D et al. ESC Guidelines for the management of acute myocardial infarction in patients presenting with ST-segment elevation. The Task Force on the management of ST-segment elevation acute myocardial infarction of the European Society of Cardiology (ESC). European Heart Journal 2012;33:2569-2619.

9. Taylor RS, Brown A, Ebrahim S et al. Exercise-based rehabilitation for patients with coronary heart disease: systematic review and metaanalysis of randomized controlled trials. Am J Med 2004;116:682-692.

10. Graham I, Atar D, Borch-Johnsen K et al. European guidelines on cardiovascular disease prevention in clinical practice: executive summary. Eur Heart J 2007;28:2375-2414.

11. Chew DP, Huynh LT, Liew D et al. Potential survival gains in the treatment of myocardial infarction. Heart 2009;95:1844-1850.

12. Ford ES, Ajani UA, Croft JB et al. Explaining the decrease in US deaths from coronary disease, 1980-2000. N Engl J Med. 2007;356:2388-2398.

13. Gąsiorowski A, Dutkiewicz J. Comprehensive rehabilitation in chronic heart failure. Ann Agric Environ Med 2013; 20(3):606-612.

14. Jankowski P, Niewada M, Bochenek A et al. Optimal model of comprehensive rehabilitation and secondary prevention. Kardiol Pol 2013;71(9):995-1003.

15. Lawler PR, Filion KB, Eisenberg MJ. Efficacy of exercise-based cardiac rehabilitation post-myocardial infarction: a systematic review and meta-analysis of randomized controlled trials. Am Heart J 2011;162 (4):571-584 e572.

16. Heran BS, Chen JM, Ebrahim S, Moxham T, Oldridge N, Rees K, Thompson DR, Taylor RS. Exercise-based cardiac rehabilitation for coronary heart disease. Cochrane Database Syst Rev 2011;7: CD001800.

17. Taylor RS, Brown A, Ebrahim S et al. Exercise-based rehabilitation for patients with coronary heart disease: systematic review and metaanalysis of randomized controlled trials. Am J Med 2004;116:682-692.

18. Hacke W, Donnan G, Fieschi C et al. Association of outcome with early stroke treatment: pooled analysis of ATLANTIS, ECASS, and NINDS rt-PA stroke trials. Lancet 2004;363:768-774.

19. WHO: International Classification of Functioning Disability and Health. Geneva, World Health Organisation, 2001.

20. Jankowski P1, Czarnecka D, Lysek R, Skrzek A et al. Secondary prevention in patients after hospitalisation due to coronary artery disease: what has changed since 2006? Kardiol Pol. 2014;72(4):355-62.

21. Giannuzzi P, Mezzani A, Saner H et al. Working Group on Cardiac Rehabilitation and Exercise Physiology. European Society of Cardiology. Physical activity for primary and secondary prevention. Position paper of the Working Group on Cardiac Rehabilitation and Exercise Physiology of the European Society of Cardiology. Eur Heart J 2003;24:1273-1278. 